\title{
Embarazo triple monocorial triamniótico: Experiencia de dos casos y revisión de la literatura
}

\author{
Javiera Valdivieso $R^{1}{ }^{1}$, Rafael Valdés $V^{2}$, Mercedes de Alvarado $R^{1}{ }^{1}$, Juan Pablo \\ Espinoza G. ${ }^{3}$, Cecilia Goity $F^{4}$, Maite Troncoso $S^{4}$, Manuel Schepeler. ${ }^{5}$, \\ Sebastián Illanes L. 4, 5,6, Horacio Figueroa D. 4, 5,'6
}

${ }^{1}$ Programa de Posgrado en Obstetricia y Ginecología Universidad de los Andes. ${ }^{2}$ Programa de Subespecialidades Médicas Medicina Materno Fetal Universidad Católica. ${ }^{3}$ Sichili Mission Hospital, Sichili, Zambia. ${ }^{4}$ Unidad de Medicina Materno Fetal, Departamento de Ginecología y Obstetricia y Biología de la Reproducción, Universidad de los Andes. 5Unidad de Medicina Materno Fetal, Clínica Dávila. Santiago, Chile. GUnidad de Medicina Materno fetal, Clínica Universidad de los Andes.

\section{RESUMEN}

Los embarazos múltiples se consideran una entidad de alto riesgo obstétrico. Su incidencia ha ido en aumento debido a la utilización de técnicas de reproducción asistida y el aumento de la edad materna. Se presentan 2 casos de embarazos triples monocoriales triamnióticos, de sexo femenino y masculino. En ambos embarazos se manifestaron complicaciones, principalmente fetales y neonatales, atribuidas a embarazos múltiples descritas en la literatura.

\section{PALABRAS CLAVES: Embarazo monocorial triamniótico, embarazo múltiple, complicaciones, embarazo triple}

\section{SUMMARY}

Multiple pregnancies are considered a high-risk obstetric entity. Their incidence has been increasing due to the use of assisted reproductive techniques and increased maternal age. Here, we describe two cases of triamniotic monochorionic triplet pregnancies, female and male respectively. Both pregnancies demonstrated complications, mainly fetal and neonatal, attributed to multiple pregnancies reported in the literature.

\section{KEY WORDS: Triamniotic monochorionic pregnancy, multiple pregnancy, complications, triple pregnancy}

\section{INTRODUCCIÓN}

La incidencia de embarazos múltiples ha aumentado en las últimas décadas, con aumento de un $40 \%$ en la tasa de embarazos gemelares y de 3 a 4 veces en embarazos múltiples de alto orden (1). Las técnicas de reproducción asistida y las gestaciones en edad materna tardías explicarían este aumento (1). Un 93\% de los embarazos triples son prematuros (edad gestacional promedio de 32-33 semanas), el $13 \%$ de los embarazos triples son prematuros extremos y su mortalidad esta aumentada 10 veces cuando se comparan con embarazos únicos (2). A pesar del aumento de su incidencia, los embarazos monocoriales triples espontáneos son muy poco frecuentes (1). Es por esto que reportamos 2 casos de embarazos triples monocoriales triamnióticos espontáneos. 


\section{Caso clínico 1}

Paciente de 23 años multípara de 1 con antecedente de cesárea anterior, derivada desde el policlínico de Alto Riesgo Obstétrico del Centro Universitario de Especialidades Médicas de San Bernardo con control de embarazo prenatal normal. Sin ecografía precoz. Se realiza ecografía que diagnostica embarazo triple, monocorial triamniótico, de 23 semanas de gestación (Figura 1). Durante el control de embarazo se realizaron las siguientes ecografías que fueron informadas sin anomalías.

- $25+3$ semanas, feto $1(F 1)$ creciendo en el percentil (p) 50, feto 2 (F2) en p 50-75, y el feto 3 (F3) en $\mathrm{p} 75$.

- 27 + 4 semanas, F1 y F2 creciendo en p50-75, y el F3 en p25-50.

- 29+4 semanas, F1 y F3 creciendo en p50-75, y F2 creciendo sobre el p90.

La evaluación cervical ecográfica fue de $36 \mathrm{~mm}$ a las $27+4$ semanas sin funnel, ni sludge y sin deformación al pujo. A las 28 semanas de gestación inicia dinámica uterina (DU) espontánea por lo que se hospitaliza y se administra un primer curso de corticoides que se repite a las 32 semanas, por la persistencia de la amenaza de parto prematuro. A las 32 semanas +3 inicia trabajo de parto por lo que se realiza cesárea de urgencia, con una adecuada profilaxis de inercia uterina con oxitocina en bolo y luego en goteo tras la extracción de los fetos y masaje uterino luego de la histerorrafia. Se obtienen 3 recién nacidos $(\mathrm{RN})$ vivos de sexo femenino; el $1^{\circ}$ y $3^{\circ}$ en podálica, el $2^{\circ}$ en cefálica. A la inspección macroscópica de la placenta impresionaba un solo macizo, con inserción velamentosa de 2 de los 3 cordones umbilicales. El puerperio inmediato fue fisiológico dándose de alta al tercer día post parto.

La gemela 1 (G1), recién nacida de pretérmino (RNPT) de 32 semanas, con peso de nacimiento (PN) de $1.705 \mathrm{~g}$, talla de $42 \mathrm{~cm}$ y Apgar 9-9 al minuto y 5 minutos de vida, con peso adecuado para la edad gestacional (AEG). Evoluciona con ictericia neonatal requiriendo fototerapia. A los 8 días de vida, presenta enterocolitis necrotizante $(E C N)$, que se trata médicamente y anemia y trombocitopenia que requirieren transfusión. Fue dada de alta a los 30 días de vida.

La gemela 2 (G2), AEG, con PN de $1.890 \mathrm{~g}$, talla $42 \mathrm{~cm}$ y Apgar de 8-9. Evoluciona en las primeras horas con un ductus arterioso persistente (DAP), que responde al manejo médico con antiprostaglandínicos. Presenta ictericia neonatal por lo que requiere fototerapia. A los 35 días de vida debuta con cuadro de ECN fulminante, que evoluciona con shock séptico refractario a tratamiento médico, fallece dentro de las primeras 24 horas de diagnóstico, sin posibilidades de intervención quirúrgica.

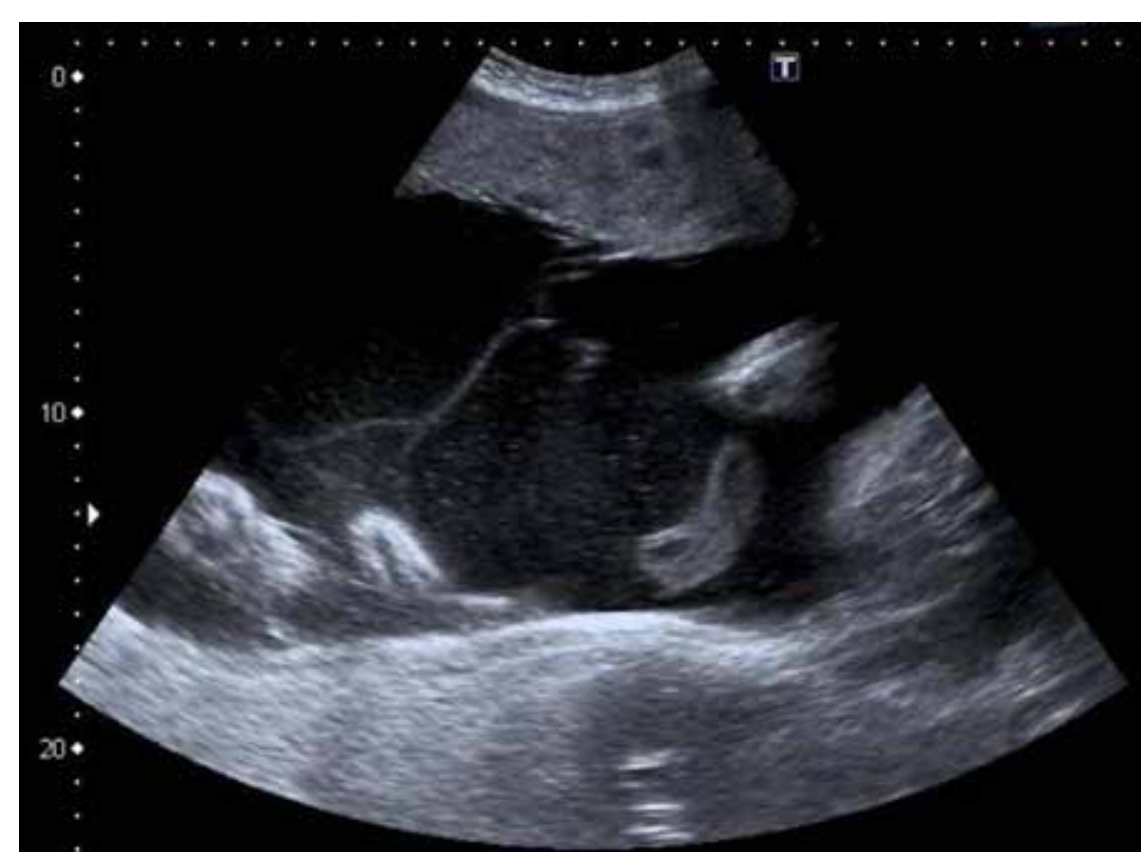

Figura 1. Ecografía de las 23 semanas donde se identifican claramente las tres membranas de división interamniótica. 
La gemela (G3) AEG, con PN de $1.595 \mathrm{~g}$, talla $42 \mathrm{~cm}$ y Apgar de 9-9. Presenta ictericia neonatal, episodios de apnea, DAP y ECN, que responde a tratamiento médico. A las 6 semanas presenta un cuadro infeccioso tratado en unidad de cuidados intensivos neonatales (UCIN). Fue dada de alta en buenas condiciones.

\section{Caso clínico 2}

Paciente de 27 años, multípara de 1 con antecedente de una cesárea. Cursando embarazo triple monocorial triamniótico de 18 semanas, diagnosticado con ecografía precoz (no se cuenta con ella) consulta en Clínica Dávila para iniciar control prenatal. Durante el control ambulatorio se diagnostica a las 27 semanas, anemia leve y diabetes gestacional que se maneja con hierro oral y metformina. A las 24 semanas de gestación inicia DU sin modificación cervical, indicándose un primer curso de corticoides, los que se repitieron a las 28 semanas por persistencia de DU aislada. A las 29 semanas +4 se realiza ecografía que muestra F1 creciendo en el p5-10 con Doppler fetal normal, F2 creciendo en el p10-25 con aumento resistencia de arterias uterinas $(\mathrm{AU})$, arteria umbilical fetal normal y vasodilatación de la arteria cerebral media fetal (ACM), y F3 creciendo en el p25-50. Cervicometría de 25 $\mathrm{mm}$ sin deformación al pujo. Se decide hospitalizar para monitorización de la unidad feto-placentaria. A las 30 semanas presenta rotura prematura de membranas (RPM) del F1 e inicio de trabajo de parto. Se realiza cesárea de urgencia y manejo profiláctico de inercia uterina con oxitocina según protocolo, sin incidentes. Puerperio inmediato fisiológico y se da de alta al tercer día posparto. Se obtienen 3 $\mathrm{RN}$ de sexo masculino de 30 semanas.

El G1, pequeño para la edad gestacional (PEG), con PN de $1.135 \mathrm{~g}$, Apgar 9-9. Evoluciona con una taquipnea transitoria (TT), que requiere oxigenoterapia. Presenta episodios de apnea del prematuro que se tratan con cafeína vía oral, ictericia neonatal que requiere fototerapia durante 3 días. Anemia e infección intrahospitalaria tratada con antibióticos sistémicos. Dado de alta a los 41 días de vida, con un peso de $2.045 \mathrm{~g}$, alimentándose por vía oral y en buenas condiciones generales.

El G2, AEG, PN de 1.285 g, Apgar 7-9, evoluciona con TT. Además presenta DAP con cierre espontáneo, apnea del prematuro que se maneja con cafeína oral, ictericia neonatal que requirió fototerapia y ECN manejada médicamente. Fue dado de alta a los 41 días de vida, en buenas condiciones generales, con un peso de $2.095 \mathrm{~g}$.

El G3, AEG con PN de 1.505 g, Apgar 8-9, cursa con enfermedad de membrana hialina que requiere ventilación mecánica por 1 día. DAP de manejo quirúrgico e ictericia neonatal que requirió fototerapia. Se da de alta a los 36 días de vida, con peso de $2.350 \mathrm{~g}$, en buenas condiciones generales.

\section{Procesamiento y análisis de las placentas}

Las placentas de ambos casos se conservaron para estudio anátomo-funcional. Los vasos fueron repletados con látex coloreado con tinta china para identificar arterias y venas. Luego fue diafanizada mediante la técnica por maceración. Se identifican estructuras con una orientación espacial definida mediante transiluminación y se observan presencia de anastomosis. En las placentas se encuentran diferencias en los tamaños placentarios de cada uno de los fetos, de los territorios vasculares y del largo de los cordones umbilicales. Se evidencian anastomosis vasculares arterio-arteriales, veno-venosas y arterio-venosas, siendo estas últimas las más frecuentes (Figuras 2 y 3 ).

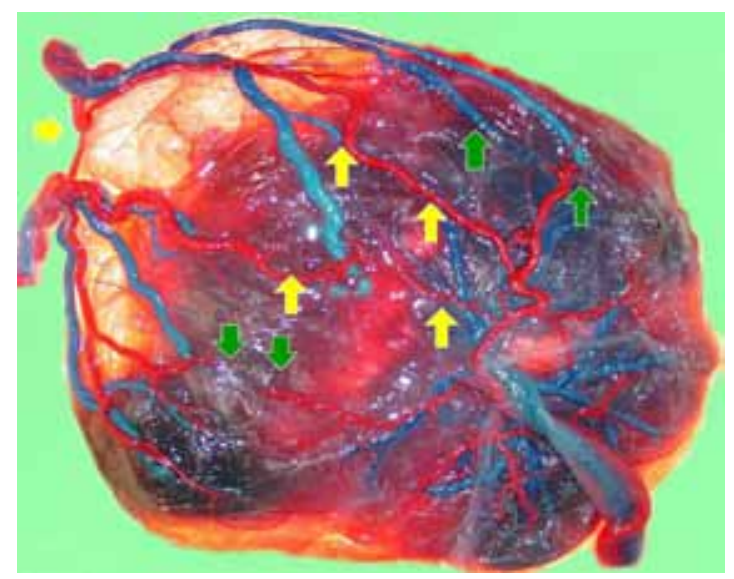

Figura 2. Placenta repletada y diafanizada. Visualización de anastomosis arterio-venosas (flecha verde) y arterio-arteriales (flecha amarilla) entre los tres lechos placentarios.

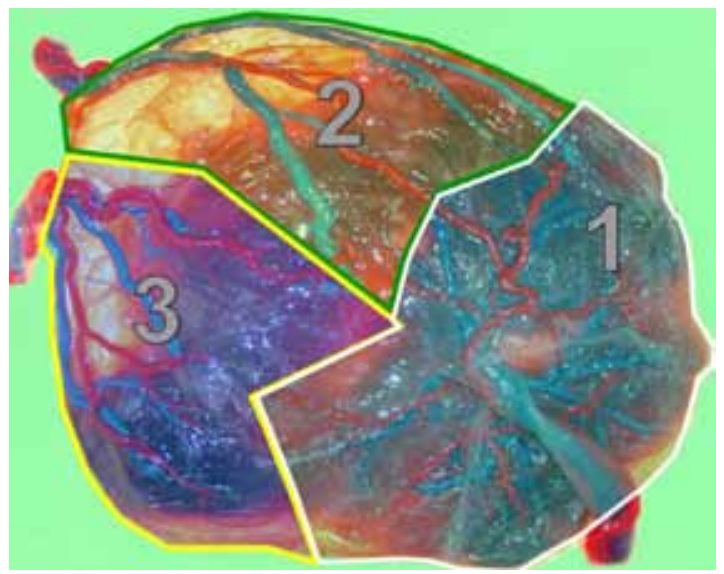

Figura 3. Mismo preparado anatómico, destacándose la asimetría de las masas placentarias. 


\section{DISCUSIÓN}

El embarazo múltiple es un embarazo de alto riesgo desde el punto de vista materno y fetal. La incidencia de estos se ha visto incrementada en la última década (3) y es responsable de una de cada 8 - 10 muertes perinatales (4). Los embarazos monocoriales tienen una tasa de morbimortalidad de $25 \%$, mayor a la de embarazos bicoriales y únicos (5).

El resultado perinatal esta dado fundamentalmente por la corionicidad. Cuando la división de los gametos ocurre dentro de los primeros 3 días desde la fecundación, da origen a la formación de placentas independientes; si esta ocurre después, el resultado será un embarazo monocorial (6). La placentación monocorial, en comparación con embarazos bicoriales, se asocia a mayor riesgo de muerte intrauterina, muerte neonatal y complicaciones de la prematuridad, especialmente discapacidad neurológica. La mayor morbimortalidad se atribuye, entre otras causas, a la presencia de anastomosis vasculares entre los gemelos (7) y la posibilidad de desarrollar síndrome de transfusión feto-fetal (STFF), secuencia de perfusión arterial reversa (TRAP) (8), restricción de crecimiento intrauterino (RCIU) con flujos diastólicos reversos o ausentes intermitentes, siendo estas complicaciones única para embarazos monocoriales. El feto muerto in útero como complicación se puede presentar en fetos bicoriales pero sin consecuencias para el otro feto.

El crecimiento fetal depende de tres aspectos: el potencial de crecimiento genético, masa placentaria y las patologías maternas concomitantes. En los casos clínicos presentados en este trabajo, los tres fetos tienen el mismo potencial de crecimiento genético y no están expuestos a ninguna patología materna asociada. La única diferencia que presentan es una distribución y masa placentaria que es evidentemente distinta el analizar las placentas. La presencia de anastomosis de diferentes tipos podrían explicar las discrepancias que se observaron en algunos Doppler donde existía vasodilatación cerebral sin aumento de resistencia de la arteria umbilical. En los embarazos monocoriales dobles la presencia de anastomosis arterio-arteriales serian la causa del flujo diastólico intermitente.

En relación a los hallazgos placentarios existieron diferencias significativas de la masa placentaria y se observó la presencia de anastomosis vasculares entre los tres fetos, sin embargo, no hubo desarrollo de STFF, y los embarazos no cursaron con las complicaciones propias del embarazo múltiple, salvo el parto prematuro.

La ecografía precoz, entre las 11 y 14 semanas de gestación, adquiere un rol protagónico para identificar y seguir oportunamente las complicacio- nes del embarazo monocorial. Se describe el signo de lamba o twin peak para determinar la corionicidad (bicorial o monocorial, respectivamente) con alta sensibilidad y especificidad $(6,9)$. Se estima que mediante una ecografía antes de las 16 semanas, determinará la corionicidad en el $100 \%$ de los embarazos múltiples (10). En estos casos, no se cuenta con las ecografías precoces, ya que fueron derivadas desde otros centros con el diagnóstico de embarazo monocorial, pero aún en la ecografía de las 23 semanas del caso 1, se evidencia el signo de twin peak. El tratamiento de las placentas evidenció la presencia de una placenta para los tres fetos, pero la prueba de mayor certeza de un embarazo monocorial es la determinación del ADN de cada RN, con secuencia idéntica entre ellos por tratarse de monocigotos.

Mientras que la tasa de embarazos triples ha aumentado, la EG promedio de nacimiento se ha mantenido estable entre las $32-33$ semanas (11). En los casos descritos la EG fue de 32 y 30 semanas, casos 1 y 2, respectivamente. La edad promedio de las gestantes en el estudio de Ricci et al fue de 29 años (3), siendo de 23 y 27 años en nuestras pacientes.

La prematuridad tiene una incidencia de hasta un $94 \%$ y el bajo PN hasta un $92 \%$, siendo estas las principales causas de mortalidad neonatal (3). Cerca del $13 \%$ de los nacimientos triples son clasificados como prematuros extremos (menores de 28 semanas) (11), los cuales presentan 15 veces más riesgo de morir dentro del primer mes de vida, asociado a una tasa de mortalidad infantil de hasta 10 a 12 veces la de un embarazo único $(3,11)$. Un $75 \%$ de los neonatos triples requieren admisión a la UCIN (11). Debido al alto riesgo de parto prematuro que presentan los embarazos gemelares, mayor aún en monocoriales y de mayor orden, se decidió administrar dos cursos de corticoides separados por 4 semanas para maduración pulmonar, a pesar de no presentar una amenaza de parto prematuro como tal en ese momento.

Las complicaciones maternas se asocian a un aumento tanto de la morbilidad de 3 a 7 veces, como a un aumento en la mortalidad materna (13). Los principales riesgo están dados por el síndrome hipertensivo del embarazo (SHE), la rotura prematura de membranas (RPM), hemorragia ante y posparto y anemia, entre otros. En el caso del embarazo triple existe un riesgo de $20 \%$ de desarrollar pre-eclampsia, $30 \%$ de anemia y $35 \%$ de hemorragia posparto (14). Dentro de esta última se encuentra la inercia uterina, con mayor frecuencia en embarazos gemelares debido a la sobre distensión uterina. A modo de prevención se utilizan uterotónicos luego de la extracción del primer gemelo e infusión oxitócica intravenosa luego del parto vaginal y de cesárea (13). 
Las complicaciones neonatales se deben a la prematuridad y al RCIU. Éstas se relacionan con la inmadurez pulmonar, infecciones, anomalías neurológicas y congénitas (3), teniendo mayor riesgo de presentar síndrome de distrés respiratorio (SDR), hemorragia intraventricular, DAP, ECN, anemia, sepsis, retinopatía del prematuro y displasia broncopulmonar (DBP). En los casos expuestos, todos los RN presentaron al menos una complicación, dado principalmente por la prematurez. Uno de ellos falleció por ECN.

\section{CONCLUSIÓN}

El embarazo triple monocorial es una entidad poco frecuente. Se asocia a una mayor morbimortalidad materna, fetal y neonatal por lo que requiere seguimiento y manejo por especialistas en un centro de alta complejidad. He ahí la importancia de la determinación de la corionicidad durante el primer trimestre, para un manejo óptimo. Dado la baja frecuencia de esta entidad, y considerando la no asociación con terapias de reproducción asistida, nos pareció relevante la publicación de estos casos clínicos.

\section{REFERENCIAS}

1. Valenzuela P, Becker J, Carvajal J. Pautas de manejo clínico de embarazos gemelares. Rev Chil Obstet Ginecol 2009;74:52-68.

2. Sumners J, Moore E, Ramsey C, Eggleston M. Transabdominal cervical cerclage in triplet pregnancies and risk of extreme prematurity and neonatal loss. J Obstet Gynaecol 2011;31:111-7.

3. Ricci P, Perucca E, Pérez C, González D, Valenzuela S, Galleguillos I. Embarazo triple: Complicaciones maternas y perinatales. Rev Chil Obstet Ginecol 2005;70:281-8.
4. Crespo R, Alvir A, Lapresta M, Andrés M, Campillos $\mathrm{J}$, Castán S. Impacto de la edad materna avanzada en las complicaciones obstétricas y perinatales de las gestaciones gemelares. Clin Invest Gin Obst 2011;39:230-5.

5. Eytan O, Har-Toov J, Almog R, Reches A, Fait G, Gull I, Wolman I, Lessing J, Loewenthal R, Gazit E, Jaffa A. Feto-feto-fetal transfusion syndrome in monozygotic monochorionictriamniotic triplets: vascular evaluation by a cast model. Placenta 2005;26:432-6.

6. Sepúlveda W, Dezerega V, Valle P, Gutiérrez J, Carstens E, Sánchez J. Determinación prenatal de la corionicidad en el embarazo gemelar. Rev Chil Ultrasonografía 1999;2:28-33.

7. Adegbite A, Ward S, Bajoria R. Perinatal outcome of spontaneously conceived triplet pregnancies in relation to chorionicity. Am J Obstet Gynecol 2005;193:1463-71.

8. Sherer D. Adverse perinatal outcome of twin pregnancies according to chorionicity: review of the literature. Am J Perinatol 2001;18:23-37.

9. Wood S, Onge R, Connors G, Elliot P. Evaluation of the twin peak or lambda sign in determining chorionicity in multiple pregnancy. Obstet Gynecol 1996;88:6-9.

10. Ministerio de Salud. Guía Perinatal 2013. SANTIAGO: MINSAL, 2013

11. Rebarber A, Roman A, Istwan N, Rhea D, Stanziano G. Prophylactic cerclage in the management of triplet pregnancies. Am J Obstet Gynecol 2005;193:1193-6.

12. Multiple gestation pregnancy. The ESHRE Capri Workshop Group. Human Reproduction 2000;15:1856-64.

13. Dudenhausen J, Maier R. Perinatal problems in multiple births. Dtsch Arztebl Int 2010;107:663.8.

14. Rao A, Sairam S, ShehataH. Obstetric complications of twin pregnancies. Best Pract Res Clin Obstet Gynaecol 2004;18:557-76.

15. Geipel A, Berg C, Katalinic A, Plath H, Hansmann M, Germer U, Gembrucha U. Prenatal diagnosis and obstetric outcomes in triplet pregnancies in relation to chorionicity. BJOG 2005;112:554-8. 